Theoretical Informatics and Applications

Theoret. Informatics Appl. 35 (2001) 331-350

\title{
TOWARDS PARAMETRIZING WORD EQUATIONS
}

\author{
H. Abdulrab ${ }^{1}$, P. Goralčí́K ${ }^{2}$ And G.S. Makanin ${ }^{3}$
}

\begin{abstract}
Classically, in order to resolve an equation $u \approx v$ over a free monoid $X^{*}$, we reduce it by a suitable family $\mathcal{F}$ of substitutions to a family of equations $u f \approx v f, f \in \mathcal{F}$, each involving less variables than $u \approx v$, and then combine solutions of $u f \approx v f$ into solutions of $u \approx v$. The problem is to get $\mathcal{F}$ in a handy parametrized form. The method we propose consists in parametrizing the path traces in the so called graph of prime equations associated to $u \approx v$. We carry out such a parametrization in the case the prime equations in the graph involve at most three variables.
\end{abstract}

Résumé. De façon classique, on résout une équation $u \approx v$ dans le monoïde libre $X^{*}$ en la réduisant par une famille convenable $\mathcal{F}$ de substitutions en une famille d'équations $u f \approx v f, f \in \mathcal{F}$, chacune en moins de variables que $u \approx v$, et ensuite en combinant des solutions des $u f \approx v f$ pour obtenir des solutions de $u \approx v$. Le problème qui se pose alors est d'obtenir $\mathcal{F}$ sous une forme commode paramétrisée. La méthode que nous proposons est basée sur la paramétrisation des traces des chemins dans le graphe des équations premières associé à $u \approx v$. Nous effectuons une telle paramétrisation dans le cas où les équations premières dans le graphe contiennent au plus trois variables.

Mathematics Subject Classification. 68R15, $20 \mathrm{M} 05$.

\section{INTRODUCTION}

We try to address the long standing and non solved problem in word equations of getting a "good presentation" of all the solutions of an equation. The early attempts in this direction aimed at how to systematically generate solutions. Lentin

\footnotetext{
Keywords and phrases: Equation, free monoid, parametrization, universal family.

${ }^{1}$ LIR/INSA de Rouen, BP. 08, 76131 Mont-Saint-Aignan Cedex, France.

${ }^{2}$ LIFAR, Université de Rouen, place E. Blondel, 76821 Mont-Saint-Aignan Cedex, France.

3 Steklov Mathematical Institute, Vavilova 42, 117966 Moscow GSP-1, Russia.
} 
[3] and Plotkin [7] presented a simple semi-algorithm to get all most general unifiers in case of solvable equations, Jaffar [1] did the same for the case of generalized equations as defined by Makanin [5].

However, what we really dream about is to describe the solutions in a closed form, by means of expressions involving variables, handy to work with.

Things would be nice indeed if the solutions of an arbitrary word equation could be represented - as it happens sometimes - by a finite number of parametric formulas, involving a finite number of constants, of word variables, and of natural parameters ranging over the set of naturals $\mathbb{N}$. As Hmelevskii [2] has pointed out, however, this type of parametrization would not work beyond the classe of the equations in at most three variables.

Still, the idea of parametrizing equations (i.e. the solutions thereof) can be saved if we admit parameters ranging not only over $\mathbb{N}$ or over a finite cartesian power of $\mathbb{N}$, but over the free monoid $\left(\mathbb{N}^{m}\right)^{*}$ of strings of $m$-tuples of naturals, or over the free monoid of strings of strings of naturals $\left(\mathbb{N}^{*}\right)^{*}$, and so on.

Typically, such parameters appear when we try to enumerate the elements of a monoid $M$ generated by a parametric family of elements of $M$. For example, if $M$ is generated by a sequence $c_{1}, \ldots, c_{m}$ of generators then it can very conveniently be parametrized by the morphism $\Phi:\left(\boldsymbol{N}^{\boldsymbol{m}}\right)^{*} \rightarrow M$ such that $\Phi\left(n_{1}, \ldots, n_{m}\right)=$ $c_{1}^{n_{1}} \cdots c_{m}^{n_{m}}$ for all $\left(n_{1}, \ldots, n_{m}\right) \in \mathbb{N}^{m}$.

This is, roughly, the idea we are going to apply, along the lines of the research programme proposed by Makanin [6], to families of elementary substitutions read out from the so called graph of prime equations associated to the equation to be solved. The graph in question will be treated piecewise, by dividing it into easily parametrisable parts called realms, in order to obtain a parametric family of substitutions taking the equation to equations involving less variables.

We demonstrate the practical usefulness of this method by applying it to the class of the equations without constants whose graphe of prime equations involves at most three variables. To confirm the method in general is a matter of further research.

In order to make the paper practically self contained, we bring all the necessary background in Sections 1 through 3. Section 4 describes the realms in the graph of prime equations in three variables and Section 5 paves the way towards parametrizing a family of substitutions efficiently reducing an equation with a three-variable prime equation.

\section{Equations And SOlutions}

The free monoid of words over a set $A$ of symbols will be denoted by $A^{*}$, its empty word by 1 , the length of $w \in A^{*}$ by $|w|$, the number of occurrences of $a \in A$ in $w$ by $|w|_{a}$, and the set of symbols occurring in $w$ by $\operatorname{alph}(w)$.

The monoid $A^{*}$ is cancellable, sut $=s v t \Rightarrow u=v$, prefix ordered, $u \leq v \Leftrightarrow \exists w \in$ $A^{*}(v=u w)$, and equidivisible, $u \leq w$ and $v \leq w \Rightarrow u \leq v$ or $u=v$ or $v \leq u$. 
A morphism $f: A^{*} \rightarrow B^{*}$ of the free monoid $A^{*}$ into another free monoid $B^{*}$ is completely determined by an assignment of word values in $B^{*}$ to the symbols of $A$, written as $(a \mapsto a f)_{a \in A}$.

In particular, any endomorphism $f$ of $A^{*}$ which leaves all but finitely many symbols fixed is called a substitution and is easily described by just giving the arrows $a \mapsto a f$ for $a \in \operatorname{Dom}(f)=\{a \in A \mid a f \neq a\}$.

The composite of a pair of morphisms $f: A^{*} \rightarrow B^{*}$ and $g: B^{*} \rightarrow C^{*}$ will be written right-hand, as $f g: A^{*} \rightarrow C^{*}$.

A morphism $g: A^{*} \rightarrow B^{*}$ divides on the left a morphism $f: A^{*} \rightarrow C^{*}$ if $f=g h$ for some morphism $h: B^{*} \rightarrow C^{*}$.

In order to be able to formulate arbitrary word equations, we fix a countably infinite set $X$ of word variables and build up the free monoid $X^{*}$ of words called terms.

Every term $u$, together with its alphabet ordered into a sequence, $\operatorname{alph}(u)=$ $\left\{x_{1}, \ldots, x_{n}\right\}$, defines a function $\varphi:\left(X^{*}\right)^{n} \rightarrow X^{*}$ such that $\varphi\left(u_{1}, \ldots, u_{n}\right)=$ $f(u)$, where $f \in \operatorname{End}\left(X^{*}\right)$ is the substitution $\left(x_{i} \mapsto u_{i}\right)_{1 \leq i \leq n}$. We have then $\varphi\left(x_{1}, \ldots, x_{n}\right)=u$, which is another way to write the term $u$.

An equation $E$ is defined as a pair $(u, v)$ of terms $u, v \in X^{*}$, often written as a single word $u \approx v$, with the special symbol $\approx$ as separator. The alphabet of $E=(u \approx v)$ is then defined as alph $(E)=\operatorname{alph}(u v)$, and the length of $E$ as $|E|=|u v|$ (the symbol $\approx$ does not count).

A pair of equations $E_{1}=\left(u_{1} \approx v_{1}\right)$ and $E_{2}=\left(u_{2} \approx v_{2}\right)$ can be multiplied into $E=E_{1} E_{2}=\left(u_{1} u_{2} \approx v_{1} v_{2}\right)$. By abuse, we then call $E_{1}$ a prefix equation and $E_{2}$ a suffix equation of $E$.

An equation $E=(u \approx v)$ is called trivial if its terms are equal (some authors say 'graphically equal' or 'coinciding'), written $u=v$. Also, more generally, an equation $E=(u \approx v)$ is called commutatively trivial (or balanced) if $|u|_{x}=|v|_{x}$ for every $x \in X$, and, semilattice trivial if $\operatorname{alph}(u)=\operatorname{alph}(v)$.

Given an arbitrary free monoid $A^{*}$, a solution of $E=(u \approx v)$ in $A^{*}$ is an arbitrary monoid morphism $f: X^{*} \rightarrow A^{*}$ taking the terms of $E$ to equal words $u f=v f$. In particular, an endomorphism $f \in \operatorname{End}\left(X^{*}\right)$ is a solution of $E$ if the image $E f=(u f \approx v f)$ of $E$ by $f$ is a trivial equation. Obviously, transposing the terms of $E=(u \approx v)$ we get of $E^{\prime}=(v \approx u)$ with exactly the same solutions.

The word variables only can take up words for values. This semantic restriction is important, because special properties of free monoids, like cancellation or equidivisibility, may enter into play. Without these strong properties only very little could be said about word equations.

One immediate consequence of the cancellation property is that for any $s, t, u, v \in$ $X^{*}$, the equations $u \approx v$ and $s u t \approx s v t$ have the same solutions. This enables us to replace, as far as solutions are concerned, any equation $E$ by its left cancelled form $l c(E)$ obtained from $E$ by cancelling its maximal trivial prefix equation. An equation $E$ will be called left cancelled if $E=l c(E)$.

Every nontrivial equation of the form $x_{1} \cdots x_{m} \approx 1$, where $x_{1}, \cdots, x_{m} \in X$, has for solutions in $A^{*}$ all morphisms $f: X^{*} \rightarrow A^{*}$ such that $x_{1} f=\ldots=x_{m} f=1$. 
This permits us to restrict our attention only to the left cancelled equations with both of its terms non-void. Every such equation $E$ is of the form $x u \approx y v$, where $u, v \in X^{*}, x, y \in X$, and $x \neq y$. The two distinct variables $x$ and $y$ are the so called pivots of $E$, written pivot $(E)=\{x, y\}$.

If $E=(u \approx v)$ is cancelled but not semilattice trivial then we can always multiply it on the right by a suitable term $t$, so as to get a left cancelled and semilattice trivial equation $F=(u t \approx v t)$. By the cancellation property again, $F$ has the same solutions as $E$.

All told, we need only consider the following more restricted set of equations.

Definition 1. The symbol $\mathcal{E}$ wil designate throughout the set of all left cancelled semilattice trivial equations over $X^{*}$.

Note that $\mathcal{E}$ is a monoid under the product of equations, with $1 \approx 1$ the identity. Here comes one of our key notions.

Definition 2. An equation $E \in \mathcal{E}$ is a prime equation if no non-trivial proper prefix of $E$ is in $\mathcal{E}$. Put otherwise, if $E=E_{1} E_{2}$ for $E_{1} \in \mathcal{E}$ and $E_{1} \neq(1 \approx 1)$ then $E_{2}=(1 \approx 1)$.

Clearly, every non-trivial equation $E \in \mathcal{E}$ has a unique prime prefix equation.

To conclude with, call $F$ similar to $E=(u \approx v)$, written $E \cong F$, if $F=(u h \approx$ $v h$ ) for some automorphism $h$ of $X^{*}$. The solutions $f$ of $F$ then correspond, in an obvious way, bijectively to the solutions $h f$ of $E$.

\section{UNIVERSALITY}

Any solution $f: X^{*} \rightarrow A^{*}$ of a given equation $E$ generates a host of new solutions of $E$ of the form $f g$, with $g: A^{*} \rightarrow B^{*}$ an arbitrary morphism of free monoids. In this way, a relatively small family of solutions of $E$ may determine all the rest of them.

Definition 3. A family $\mathcal{F}$ of endomorphisms of the free monoid $X^{*}$ is called universal for an equation $E$ if every solution of $E$ is divided on the left by a member of $\mathcal{F}$. If, moreover, every $f \in \mathcal{F}$ is a solution of $E$ then $\mathcal{F}$ is called a universal family of solutions of $E$ and the equation $E$ is said to be universally solved by $\mathcal{F}$.

Remark. A universal family of solutions of $E$ is called a general solution of $E$ if it is minimal, in the sense that no proper subfamily of $\mathcal{F}$ is universal. However, we are not concerned here with this property.

Here are some examples of universal solutions.

Proposition 1. Every equation $x \approx w$ such that $x \in X \backslash \operatorname{alph}(w)$ is universally solved by the substitution $x \mapsto w$.

Proposition 2. Every nontrivial equation $x^{m} \approx x^{n}$, with $x \in V$ and $m \neq n$, is universally solved by the substitution $x \mapsto 1$. 
Proposition 3. The equation $E=(x z \approx z y)$ is universally solved by the union of

and

$$
\left\{\left(x \mapsto z x, y \mapsto x z, z \mapsto(z x)^{m} z\right) \mid m \geq 0\right\}
$$

$$
\left\{\left(x \mapsto x^{r}, y \mapsto x^{r}, z \mapsto x^{s}\right) \mid r, s \geq 0\right\} .
$$

Proposition 4 (Hmelevskiı). The equation $E=(y x z \approx z y x)$ is universally solved by

$$
\left\{\left(x \mapsto(x y)^{i} x, y \mapsto(y x)^{j} y, z \mapsto(y x)^{k}\right) \mid i, j, k \geq 0\right\}
$$

together with

$$
\left\{\left(x \mapsto 1, y \mapsto 1, z \mapsto x^{k}\right) \mid k \geq 0\right\} .
$$

Let us now examine the notion of universal family of endomorphisms. If well chosen, a universal family $\mathcal{F}$ may take $E$ to a family of equations $E \mathcal{F}=\{E f \mid$ $f \in \mathcal{F}\}$ which are easier to solve (e.g. involving less variables or in some other way simpler). Moreover, this kind of reduction can be iterated, according to the following mechanism of cascade composition.

Proposition 5. Let $\mathcal{F} \subseteq \operatorname{End}\left(X^{*}\right)$ be a universal family of endomorphisms for $E$, and, for every $f \in \mathcal{F}$, let $\mathcal{G}_{f}$ be a universal family of endomorphisms for $E f$. Then the family

$$
\mathcal{G}=\bigcup\left\{f \mathcal{G}_{f} \mid f \in \mathcal{F}\right\}
$$

is a universal family of endomorphisms for $E$. If, moreover, every $\mathcal{G}_{f}$ is a universal family of solutions of $E f$ then $\mathcal{G}$ is a universal family of solutions of $E$.

Thus, finding a suitable universal family of endomorphisms for $E$ may be seen as a simpler problem than resolution, a step towards a universal family of solutions of $E$.

The following well known result ( $c f$. [4]), though of limited practical use, nicely illustrates the idea of reduction via universal family.

Theorem 2.1 (Defect theorem). Let $E=(u, v)$ be a non trivial equation with $\operatorname{alph}(E)=\left\{x_{1}, \ldots, x_{n}\right\}$. Then the family $\mathcal{F}$ of all substitutions $f$ such that $\operatorname{Dom}(f)=\operatorname{alph}(E)$ and alph $(x f) \subseteq\left\{x_{1}, \ldots, x_{n-1}\right\}$ for $x \in \operatorname{alph}(E)$ is universal for $E$.

The family $\mathcal{F}$ of this theorem becomes intractable as soon as $E$ involves three or more variables. However, if $\operatorname{alph}(E)=\{x, y\}$ then the family is

$$
\mathcal{F}=\left\{f_{i j}=\left(x \mapsto x^{i}, y \mapsto x^{j}\right) \mid i, j \geq 0\right\}
$$

and its members take $E$ to

$$
E f_{i j}=\left(x^{i|u|_{x}+j|u|_{y}} \approx x^{i|v|_{x}+j|v|_{y}}\right),
$$

which is trivial if and only if

$$
i\left(|u|_{x}-|v|_{x}\right)=j\left(|u|_{y}-|v|_{y}\right) .
$$


Proposition 6. Every equation $E=(u \approx v)$ with alph $(E)=\{x, y\}$ is universally solved by the family of substitutions

$$
\left\{\left(x \mapsto x^{i}, y \mapsto x^{j}\right) \mid i\left(|u|_{x}-|v|_{x}\right)=j\left(|u|_{y}-|v|_{y}\right), i, j \geq 0\right\} .
$$

For the equations involving more than two variables, however, more elaborate methods are needed.

\section{Pivotings And Deletions, Realms}

A classical way to treat an equation $E \in \mathcal{E}$ is to transform it into $l c(E f)$ by either a pivoting $f=(x \mapsto y x)$ or by a deletion $f=(x \mapsto 1)$, where $x, y \in \operatorname{pivot}(E)$.

The pivotings and deletions represented by labelled arrows $E \stackrel{f}{\rightarrow} F$ from $E$ to $F=l c(E f)$ structure $\mathcal{E}$ into a graph that will be denoted by $\mathcal{E}_{P D}$. The subgraph on $\mathcal{E}$ defined by the pivotings only will be denoted by $\mathcal{E}_{P}$.

In $\mathcal{E}_{P D}$, exactly four labelled arrows issue from every non-trivial equation $E \in \mathcal{E}$ with $\operatorname{pivot}(E)=\{x, y\}$. Their four labels

$$
\{(x \mapsto y x),(y \mapsto x y),(x \mapsto 1),(y \mapsto 1)\}
$$

may without danger be abbreviated to $y x, x y, x, y$, respectively.

The four substitutions labelling the arrows issued from $E$ turn out to be a universal family for $E$. Moreover, dividing out a solution $g: X^{*} \rightarrow A^{*}$ of $E$ by a member $f$ of the family may take it to a solution of $E f$ reduced in terms of the norm defined by

$$
\|g\|_{E}=\sum_{x \in \operatorname{alph}(E)}|x g|+|\operatorname{alph}(E)| .
$$

Proposition 7. For every non-trivial equation $E \in \mathcal{E}$ and for every solution $g$ : $X^{*} \rightarrow A^{*}$ of $E$, there exists an arrow $E \stackrel{f}{\rightarrow} F$ and a solution $h: X^{*} \rightarrow A^{*}$ of $F$ such that $g=f h$ and $\|h\|_{F}<\|g\|_{E}$.

Proof. If $E=(x u \approx y v)$ then $x g$ and $y g$ are prefixes of $(x u) g=(y v) g$, thus by equidivisibility, one is a prefix of the other one. Assume that $x g=(y g) w$, for some $w \in A^{*}$. Now, if $w \neq 1 \neq y g$ then we have $g=f h$ for $f=(x \mapsto y x)$ and $h: X^{*} \rightarrow A^{*}$ such that $x h=w$ and $z h=z g$ for $z \neq x$; if $w=1$ or $y g=1$ then $g=f h$ for $f=(y \mapsto 1)$ and $h=g$.

In both cases, $h$ is a solution of $E f$, thus also of $F=l c(E f)$. Moreover, we have $\|h\|_{F}<\|g\|_{E}$, because in the first case $|x h|=|x g|-|y g|$ and $\operatorname{alph}(F)=\operatorname{alph}(E)$, and in the second $\operatorname{alph}(F)=\operatorname{alph}(E) \backslash\{y\}$.

Every path

$$
E_{0} \stackrel{f_{1}}{\rightarrow} E_{1} \stackrel{f_{2}}{\longrightarrow} \ldots \stackrel{f_{k}}{\rightarrow} E_{k}
$$

in $\mathcal{E}_{P D}$ defines a composite substitution $f_{1} \cdots f_{k}$ (the identity if $k=0$ ), called the trace of the path. 
Theorem 3.1. For any equation $E \in \mathcal{E}$, the traces of the paths from $E$ to the trivial equation $1 \approx 1$ form a universal family of solutions of $E$.

Proof. Clearly, every trace of a path from $E$ to $1 \approx 1$ is a solution of $E$. To prove the universality of the family, we proceed by the induction on norm.

The assertion is true for $1 \approx 1$. Consider a non-trivial $E \in \mathcal{E}$ and a solution $g: X^{*} \rightarrow A^{*}$ of $E$, assuming the assertion true for all $F \in \mathcal{E}$ admitting a solution $h: X^{*} \rightarrow A^{*}$ such that $\|h\|_{F}<\|g\|_{E}$. The arrow $E \stackrel{f}{\rightarrow} F$ and the solution $h$ of $F$ of Proposition 7 is such. By the hypothesis, there is a path from $F$ to $1 \approx 1$ whose trace $f_{1} \cdots f_{k}$ divides $h$, say, $h=f_{1} \cdots f_{k} \cdot h^{\prime}$. Now, we have $g=f h$, whence $g=f \cdot f_{1} \cdots f_{k} \cdot h^{\prime}$ and $f \cdot f_{1} \cdots f_{k}$ is a trace of a path from $E$ to $1 \approx 1$.

We will explore the graph $\mathcal{E}_{P D}$ with the aid of the following graph-theoretical notion.

Definition 4. A subgraph $\mathcal{R}$ of a graph $\mathcal{G}$ is a realm in $\mathcal{G}$ if

1. $\mathcal{R}$ is a union of strongly connected components of $\mathcal{G}$;

2. $\mathcal{R}$ contains a vertex from which all vertices of $\mathcal{R}$ are reachable in $\mathcal{R} ;$

3. and every path in $\mathcal{G}$ conecting two vertices of $\mathcal{R}$ is entirely contained in $\mathcal{R}$.

An escape arrow from $\mathcal{R}$ is an arrow from a vertex in $\mathcal{R}$ to a vertex outside $\mathcal{R}$.

An escape path from $\mathcal{R}$ starts at a vertex in $\mathcal{R}$ and is terminated by an escape arrow from $\mathcal{R}$.

The following is a straightforward corollary of Theorem 3.1.

Proposition 8. Let $\mathcal{R}$ be a realm in $\mathcal{E}_{P D}$ such that $E \in \mathcal{R}$ and $T \notin \mathcal{R}$. Then the traces of the escape paths from $\mathcal{R}$ starting at $E$ form a universal family of substitutions for $E$.

Some realms in $\mathcal{E}_{P D}$ can be defined in terms of pivotings only.

Proposition 9. Every realm $\mathcal{R}$ in $\mathcal{E}_{P}$ is a realm in $\mathcal{E}_{P D}$.

Proof. If $\mathcal{R}$ is a realm in $\mathcal{E}_{P}$ then, for some $E \in \mathcal{R}$, all $F \in \mathcal{R}$ are accessible by a path of pivotings from $E$, whence $\operatorname{alph}(F)=\operatorname{alph}(E)$. But then every path in $\mathcal{E}_{P}$ connecting two vertices of $\mathcal{R}$ consists of pivotings only, hence all its vertices belong to $\mathcal{R}$.

The equations of the graph of pivotings $\mathcal{E}_{P}$ can be divided into the classes $[E]$ of equations having the same prime prefix equation as $E$. We will call $[E]$ the cap of $E$ and write $[E]=u \square v$ when the prime prefix equation of $E$ is $u \approx v$. The variables in alph(uv) then will be refered to as the cap variables.

Proposition 10. If $F \in[E]$ then $\operatorname{pivot}(F)=\operatorname{pivot}(E)$ and $[l c(F f)]=[l c(E f)]$ for any pivoting $f$ defined at $E$.

Put otherwise, we have a factorization $E \mapsto[E]$ of the graph of pivotings $\mathcal{E}_{P}$ onto the graph of cap pivotings $\left[\mathcal{E}_{P}\right]$, defined by the arrows $[E] \stackrel{f}{\rightarrow}[F]$ for $E \stackrel{f}{\rightarrow} F$ in $\mathcal{E}_{P}$. 
The use we are going to make of this factorization appears clearly in the following proposition:

Proposition 11. For any realm $\mathcal{S}$ in $\left[\mathcal{E}_{P}\right]$, its inverse image under the factorization, $\mathcal{R}=\left\{F \in \mathcal{E}_{P} \mid[F] \in \mathcal{S}\right\}$, is a realm in $\mathcal{E}_{P D}$. For $E \in \mathcal{R}$, the traces of the escape paths from $\mathcal{R}$ starting at $E$ are exactly the traces of the escape paths from $\mathcal{S}$ starting at $[E]$ plus the traces of the paths from $[E]$ to all $[F] \in \mathcal{S}$ composed with a deletion defined at $F$.

In this way, the search for universal families may essentially be carried out within the quotient graph $\left[\mathcal{E}_{P}\right]$, with a particular attention to its strong components as building blocs of realms and to the way they are related by the escape arrows.

The task is, however, for the whole graph $\left[\mathcal{E}_{P}\right]$, very technical and difficult. For this reason we will have it carried out only for a relatively small portion of $\left[\mathcal{E}_{P}\right]$. Those who envisage to build a cathedral are adwised first to try to construct a cage for birds.

\section{Caps in three variables}

In this section we will apply the methods we have developed so far to the caps involving at most three cap variables. Given such a cap, we can always rename the three variables into $x, y$ and $z$. Moreover, permuting the latter we can give every cap a more restricted form.

Proposition 12. Every cap $[E]$ in two variables is isomorphic to one of the following three forms:

$$
x y \square y x, x^{m+2} y \square y x, x^{m+2} y \square y^{n+2} x .
$$

Every cap $[F]$ in three variables is isomorphic to a cap of one of the following two forms:

$$
\begin{array}{ll}
x \varphi(x, y) z \square y \psi(y, z) x & (y-c a p), \\
x \varphi(x, y) z \square z \chi(y, z) x \quad(z-c a p),
\end{array}
$$

where $\varphi$ and $\chi$ contain $y$ and $\psi$ contains $z$.

Proof. Renaming the variables, we can bring the left term of $[F]$ to the form $x \varphi(x, y) z$. The right term then has one of the three forms $y \psi(y, z) x, z \chi(y, z) x$, or $z \tau(x, z) y$. However, the cap $x \varphi(x, y) z \square z \tau(x, z) y$ can be taken to $x \tau(y, x) z \square y \varphi$ $(y, z) x$ by $(x \mapsto y \mapsto z \mapsto x)$.

Our next task will be to subdivide the caps of the form specified by the above proposition into a well founded escape hierarchy of particular realms admitting a straightforward path enumeration. Each one of the realm forms we will have to introduce will be described as a function $\mathcal{R}_{\alpha}(m, n, \ldots, \varphi, \psi, \ldots)$ indexed by an ordinal number $\alpha$ and possibly depending on some natural parameters $m, n, \ldots$ ranging over the non-negative integers, and, on some word parameters $\varphi, \psi, \ldots$ ranging over $\{x, y, z\}^{*}$. For every index $\alpha$, the easy task of checking up the graph 
structure of the $\alpha$-th realm will be left to the reader. The only difficulty one may have - that of justifying the escape arrows - is resolved by noting that the alledged escape arrows from $\mathcal{R}_{\alpha}$ always go to realms of strictly less indices and that every $\mathcal{R}_{\beta}$ with $\beta<\alpha$ is disjoint from $\mathcal{R}_{\alpha}$. Here come the realms.

Proposition 13. $\mathcal{R}_{0}=\mathcal{C}(x y \square y x)$ is formed by an $x$-loop and an $y$-loop, hence it has no escape arrow.

$\mathcal{R}_{1}(m)=\mathcal{C}\left(x^{m+2} y \square y x\right)$ is an $y$-loop escaped by

$$
x^{m+2} y \square y x \stackrel{y x}{\longrightarrow} x y \square y x \in \mathcal{R}_{0} .
$$

$\mathcal{R}_{2}(m, n)=\left\{x^{m+2} y \square y^{n+2} x\right\}$ is a singleton escaped by

$$
\begin{gathered}
x^{m+2} y \square y^{n+2} x \stackrel{y x}{\longrightarrow} x y \square y^{n+2} x \in \mathcal{R}_{1}(x \leftrightarrow y), \\
x^{m+2} y \square y^{n+2} x \stackrel{x y}{\longrightarrow} x^{m+2} y \square y x \in \mathcal{R}_{1} .
\end{gathered}
$$

Proposition 14. $\mathcal{R}_{3}=\mathcal{C}(x y z \square y z x)$ is formed by the two cycles

$$
\begin{aligned}
& x y z \square z y x \stackrel{z x}{\longrightarrow} x y z \square y z x \stackrel{y x}{\longrightarrow} x y z \square z y x, \\
& x y z \square z y x \stackrel{x z}{\longrightarrow} y x z \square z y x \stackrel{y z}{\longrightarrow} x y z \square z y x,
\end{aligned}
$$

meeting at $x y z \square z y x$, and by the two $y$-loops

$$
x y z \square y z x \stackrel{x y}{\longrightarrow} x y z \square y z x, y x z \square z y x \stackrel{z y}{\longrightarrow} y x z \square z y x .
$$

There are no escape arrows.

Proposition 15. $\mathcal{R}_{4}=\mathcal{C}(x y y z \square y z x)$ is formed by the infinite $y$-path

$$
\left\{x y x^{k} y z \square y z x \mid k \geq 0\right\},
$$

connected by the infinitely many x-paths

$$
\left\{x y x^{k} y z \square y z x \stackrel{y x}{\longrightarrow} x y(y x)^{k} y z \square z y x \stackrel{z x}{\longrightarrow} x y y z \square y z x \mid k \geq 0\right\}
$$

to the infinitely many $z$-cycles

$$
\left\{\sigma(x, y) z \square z y x \mid \sigma \sim(x y)^{k+1} y\right\}, k \geq 0 .
$$

The escape arrows start at the $z$-caps distinct from $x y(y x)^{k} y z \square z y x$, and are of the form

$$
\begin{gathered}
y y \cdots \square z y x \stackrel{z y}{\longrightarrow} y z \square z y \in \mathcal{R}_{0}(x \leftrightarrow z), \\
x y x \cdots \square z y x \stackrel{z x}{\longrightarrow} x y z \square y z x \in \mathcal{R}_{3}, \\
y x y \cdots \square z y x \stackrel{z y}{\longrightarrow} y x z \square z y x \in \mathcal{R}_{3} .
\end{gathered}
$$


Proposition 16. $\mathcal{R}_{5}=\mathcal{C}(x y z \square y z z x)$ is formed by the two cycles

$$
\begin{aligned}
& x y z \square y z z x \stackrel{y x}{\longrightarrow} x y z \square z z y x \stackrel{z x}{\longrightarrow} x y z \square z y z x \stackrel{z x}{\longrightarrow} x y z \square y z z x, \\
& y x z \square x z z y \stackrel{x y}{\longrightarrow} y x z \square z z x y \stackrel{z y}{\longrightarrow} y x z \square z x z y \stackrel{z y}{\longrightarrow} y x z \square x z z y,
\end{aligned}
$$

the two cross-arrows

$$
x y z \square z z y x \stackrel{x z}{\longrightarrow} y x z \square z x z y, y x z \square z z x y \stackrel{y z}{\longrightarrow} x y z \square z y z x,
$$

and the two loops

$$
x y z \square y z z x \stackrel{x y}{\longrightarrow} x y z \square y z z x, x y z \square y z z x \stackrel{y x}{\longrightarrow} x y z \square y z z x .
$$

It is escaped by

$$
\begin{gathered}
x y z \square z y z x \stackrel{x z}{\longrightarrow} y x z \square z y x \in \mathcal{R}_{3}, \\
y x z \square z x z y \stackrel{y z}{\longrightarrow} x y z \square z x y \in \mathcal{R}_{3}(x \mapsto z \mapsto y \mapsto x) .
\end{gathered}
$$

Proposition 17. Let $\sigma=\sigma(y, z)$ be a word not conjugate to $(y z)^{k+2}$ and such that $|\sigma|_{y},|\sigma|_{z} \geq 2$, but $y^{3}, z^{3}$ are not factors of $\sigma^{2}$. Then $\mathcal{R}_{6}(\sigma)=\mathcal{C}(x y z \square \sigma(y, z) x)$ is an $x$-cycle escaped by the arrows of the form

$$
\begin{gathered}
x y z \square z z y \cdots x \stackrel{x z}{\longrightarrow} y x z \square z x z y \in \mathcal{R}_{5}(x \leftrightarrow y), \\
x y z \square z y z \cdots x \stackrel{x z}{\longrightarrow} y x z \square z y x \in \mathcal{R}_{3}, \\
x y z \square z y y z \cdots x \stackrel{x z}{\longrightarrow} y x z \square z y y x \in \mathcal{R}_{4}(x \leftrightarrow z), \\
x y z \square y^{2} z \cdots x \stackrel{x y}{\longrightarrow} x y \square y x \in \mathcal{R}_{0}, \\
x y z \square y z y \cdots x \stackrel{x y}{\longrightarrow} x y z \square y z x \in \mathcal{R}_{3}, \\
x y z \square y z z y \cdots x \stackrel{x y}{\longrightarrow} x y z \square y z z x \in \mathcal{R}_{5} .
\end{gathered}
$$

Note that in Proposition 17 the conditions stipulated for $\sigma$ make $\mathcal{R}_{6}(\sigma)$ disjoint from $\mathcal{R}_{4}(x \leftrightarrow z)$.

Proposition 18. $\mathcal{R}_{7}=\mathcal{C}(x y y z \square y z z x)$ is an infinite realm divided into two disjoint isomorphic parts, $\mathcal{H}$ and $\mathcal{H}(y \leftrightarrow z)$. The part $\mathcal{H}$ is formed by the $x$-arrow

$$
x y y z \square z y z x \stackrel{z x}{\longrightarrow} x y y z \square y z z x,
$$

by the infinite $y$-path

$$
\left\{x y x^{k} y z \square y z z x, k \geq 0\right\},
$$

by the infinitely many $x$-paths

$$
\left\{x y x^{k} y z \square y z z x \stackrel{y x}{\longrightarrow} x y(y x)^{k} y z \square z z y x \stackrel{z x}{\longrightarrow} x y y z \square z y z x, k \geq 0\right\},
$$

and by the infinitely many z-arrows

$$
\left\{x y(y x)^{k} y z \square z z y x \stackrel{x z}{\longrightarrow} y(y x)^{k+1} z \square z x z y, k \geq 0\right\} .
$$


The two parts of $\mathcal{R}_{7}$ are cross-connected by the infinitely many arrows

$$
\left\{y(y x)^{k+1} z \square z x z y \stackrel{z y}{\longrightarrow} y z y x \square x z z y, k \geq 0\right\},
$$

and by their $(y \leftrightarrow z)$-images

$$
\left\{y x y z \square z(z x)^{k+1} y \stackrel{y z}{\longrightarrow} x y y z \square z y z x, k \geq 0\right\} .
$$

$\mathcal{R}_{7}$ is escaped by the arrows

$$
\begin{gathered}
x y y z \square z y z x \stackrel{x z}{\longrightarrow} y y x z \square z y x \in \mathcal{R}_{4}, \\
y y x z \square z x z y \stackrel{y z}{\longrightarrow} y x y z \square z x y \in \mathcal{R}_{5}(x \mapsto z \mapsto y \mapsto x), \\
y(y x)^{k+2} z \square z x z y \stackrel{y z}{\longrightarrow}(y x)^{k+2} y z \square z x y \in \mathcal{R}_{6}(x \mapsto z \mapsto y \mapsto x),
\end{gathered}
$$

for $k \geq 0$, and by their $(y \leftrightarrow z)$-images.

Proposition 19. $\mathcal{R}_{8}(n)=\mathcal{C}\left(x y z \square y z^{n+3} x\right)$ is formed by a $y$-loop at $x y z \square y z^{n+3} x$ and by an $x$-cycle

$$
\left\{x y z \square z^{i} y z^{s-k+3} x \mid 0 \leq i \leq n+3\right\} .
$$

It is escaped by

$$
\begin{gathered}
x y z \square z^{i+3} y z^{n-i} x \stackrel{x z}{\longrightarrow} y x z \square(z x)^{i+2} z y \in \mathcal{R}_{6}(x \leftrightarrow y), 0 \leq i \leq n, \\
x y z \square z^{2} y z^{n+1} x \stackrel{x z}{\longrightarrow} y x z \square z x z y \in \mathcal{R}_{5}(x \leftrightarrow y), \\
x y z \square z y z^{n+2} x \stackrel{x z}{\longrightarrow} y x z \square z y x \in \mathcal{R}_{3} .
\end{gathered}
$$

Proposition 20. Let $\sigma=\sigma(y, z)$ be a word such that $|\sigma|_{y},|\sigma|_{z} \geq 2$ and $y^{3}$ or $z^{3}$ is a factor of $\sigma^{2}$. Then $\mathcal{R}_{9}(\sigma)=\mathcal{C}(x y z \square \sigma(y, z) x)$ is an $x$-cycle escaped by the arrows of the form

$$
\begin{gathered}
x y z \square z^{r+3} y \cdots x \stackrel{x z}{\longrightarrow} y x z \square z(x z)^{r+2} y \in \mathcal{R}_{6}(x \leftrightarrow y), \\
x y z \square z z y \cdots x \stackrel{x z}{\longrightarrow} y x z \square z x z y \in \mathcal{R}_{5}(x \leftrightarrow y), \\
x y z \square z y z \cdots x \stackrel{x z}{\longrightarrow} y x z \square z y x \in \mathcal{R}_{3}, \\
x y z \square z y y z \cdots x \stackrel{x z}{\longrightarrow} y x z \square z y y x \in \mathcal{R}_{4}(x \leftrightarrow z), \\
x y z \square z y^{s+3} z \cdots x \stackrel{x z}{\longrightarrow} y x z \square z y^{s+3} x \in \mathcal{R}_{7}, \\
x y z \square y^{s+2} z \cdots x \stackrel{x y}{\longrightarrow} x y \square y x \in \mathcal{R}_{0}, \\
x y z \square y z y \cdots x \stackrel{x y}{\longrightarrow} x y z \square y z x \in \mathcal{R}_{3}, \\
x y z \square y z z y \cdots x \stackrel{x y}{\longrightarrow} x y z \square y z z x \in \mathcal{R}_{5}, \\
x y z \square y z^{r+3} y \cdots x \stackrel{x y}{\longrightarrow} x y z \square y z^{r+3} x \in \mathcal{R}_{8} .
\end{gathered}
$$


Proposition 21. Every cap of the form $x^{m+1} y \varphi(x, y) z \square y z x$, where if $m=0$ then $\varphi$ contains $x$ but is not of the form $x^{k+1} y$, defines the realm

$$
\mathcal{R}_{10}(\varphi)=\left\{x^{m+1} \varphi\left(x, x^{k} y\right) z \square y z x \mid k \geq 0\right\}
$$

which is an infinite $y$-path if $\varphi$ contains $y$ and a $y$-loop otherwise, and is escaped by

$$
x^{m+1} y \varphi\left(x, x^{k} y\right) z \square y z x \stackrel{y x}{\longrightarrow}(x y)^{m+1} \varphi\left(y x,(y x)^{k} y\right) z \square z y x
$$

to $\left(\mathcal{R}_{6} \cup \mathcal{R}_{9}\right)(x \leftrightarrow z)$.

Note that in Proposition 21 the conditions stipulated for $\varphi$ make $\mathcal{R}_{10}(\varphi)$ disjoint from $\mathcal{R}_{4}$.

Proposition 22. $\mathcal{R}_{11}(r, s)=\mathcal{C}\left(x y^{r+1} z \square z y^{s+1} x\right)$, where $r \leq s$ and $r+s \geq 2$, is a union of an $x$-cycle and of an $z$-cycle meeting at $x y^{r+1} z \square z y^{s+1} x$, escaped by

$$
\begin{gathered}
x y^{r+1} z \square y^{i+2} z y^{s-i-1} x \stackrel{x y}{\longrightarrow} x y \square y x \in \mathcal{R}_{0}, 0 \leq i \leq s-1, \\
x y^{r+1} z \square y z y^{s} x \stackrel{x y}{\longrightarrow}(x y)^{r+3} z \square y z x \in \mathcal{R}_{3} \cup \mathcal{R}_{4} \cup \mathcal{R}_{10},
\end{gathered}
$$

and by the arrows obtained from these by transposing $x$ and $z$ as well as $r$ and $s$.

Note that in Proposition 22 the conditions stipulated for $r, s$ make $\mathcal{R}_{11}(r, s)$ disjoint from $\mathcal{R}_{4}(x \leftrightarrow z)$.

Proposition 23. $\mathcal{R}_{12}(\varphi, \psi)=\{x y x \varphi(x, y) z \square z y z \psi(y, z) x\}$ is a singleton realm escaped by

$$
x y x \varphi(x, y) z \square z y z \psi(y, z) x \stackrel{z x}{\longrightarrow} x y z \square y z \psi(y, z) z x \in \mathcal{R}_{5} \cup \mathcal{R}_{6} \cup \mathcal{R}_{9}
$$

and by its $(x \leftrightarrow z)$-image (with $\varphi$ and $\psi$ exchanged).

Proposition 24. $\mathcal{R}_{13}(\psi)=\{x y x z \square z z y \psi(y, z) x\}$ is a singleton realm escaped by

$$
\begin{gathered}
x y x z \square z z y \psi(y, z) x \stackrel{z x}{\longrightarrow} x y z \square z y \psi(y, z) z x \in \mathcal{R}_{5} \cup \mathcal{R}_{6} \cup \mathcal{R}_{8} \cup \mathcal{R}_{9}, \\
x y x z \square z z y \psi(y, z) x \stackrel{x z}{\longrightarrow} y x x z \square z x z y \in \mathcal{R}_{7}(x \leftrightarrow y) .
\end{gathered}
$$

Proposition 25. $\mathcal{R}_{14}(m, \tau, \sigma)=\left\{x^{m+2} y \tau(x, y) z \square z y z y \sigma(y, z) x\right\}$ is a singleton realm escaped by

$$
x^{m+2} y \tau(x, y) z \square z y z y \sigma(y, z) x \stackrel{x z}{\longrightarrow} x^{m+1} y \tau(x, y) x z \square z y x
$$

to $\left(\mathcal{R}_{6} \cup \mathcal{R}_{9}\right)(x \leftrightarrow z)$ if $\tau$ contains $y$, else to $\left(\mathcal{R}_{5} \cup \mathcal{R}_{8}\right)(x \leftrightarrow z)$, and by

$$
x^{m+2} y \tau(x, y) z \square z y z y \sigma(y, z) x \stackrel{z x}{\longrightarrow} x(z x)^{m+1} y \square y z y \sigma(y, z) z x
$$

to $\mathcal{R}_{12}(y \leftrightarrow z)$. 
Proposition 26. $\mathcal{R}_{15}(s)=\mathcal{C}\left(x y y z \square y z^{s+3} x\right)$ is a realm formed by an $x$-cycle,

$$
\left\{x y y z \square z^{j} y z^{s-j+3} x \mid 0 \leq j \leq s+3\right\},
$$

by an infinite $y$-path,

$$
\left\{x y x^{r} y z \square y z^{s+3} x \mid r \geq 0\right\},
$$

and by infinitely many $x$-paths

$$
\left\{x y x^{r+1} y z \square y z^{s+3} x \stackrel{y x}{\longrightarrow} x y(y x)^{r+1} y z \square z^{s+3} y x \stackrel{z x}{\longrightarrow} x y y z \square z^{s+2} y z x \mid r \geq 0\right\} .
$$

$\mathcal{R}_{15}(s)$ is escaped by the $z$-arrows from its z-caps, namely,

$$
\begin{gathered}
x y y z \square z y z^{s+2} x \stackrel{x z}{\longrightarrow} y y x z \square z y x \in \mathcal{R}_{4}, \\
x y y z \square z^{2} y z^{s+1} x \stackrel{x z}{\longrightarrow} y y x z \square z x y \in \mathcal{R}_{13}(x \leftrightarrow y), \\
x y y z \square z^{k+3} y z^{s-k} x \stackrel{x z}{\longrightarrow} y y x z \square z(x z)^{k+2} y \in \mathcal{R}_{12}(x \leftrightarrow y), 0 \leq k \leq s, \\
x y(y x)^{r+1} y z \square z^{s+3} y x \stackrel{x z}{\longrightarrow} y(y x)^{r+2} z \square z(x z)^{s+2} y \in \mathcal{R}_{14}(x \leftrightarrow y) .
\end{gathered}
$$

Proposition 27. $\mathcal{R}_{16}(\sigma)=\mathcal{C}(x y y z \square \sigma(y, z) x)$, where $|\sigma|_{y},|\sigma|_{z} \geq 2$, is an $x$-cycle escaped by (not necessarily all) arrows of the form

$$
\begin{gathered}
x y y z \square z^{r+3} y \cdots x \stackrel{x z}{\longrightarrow} y y x z \square z(x z)^{r+2} y \in \mathcal{R}_{14}(x \leftrightarrow y), \\
x y y z \square z z y \cdots x \stackrel{x z}{\longrightarrow} y y x z \square z x z y \in \mathcal{R}_{13}(x \leftrightarrow y), \\
x y y z \square z y z \cdots x \stackrel{x z}{\longrightarrow} y y x z \square z y x \in \mathcal{R}_{4}, \\
x y y z \square z y^{s+2} z \cdots x \stackrel{x z}{\longrightarrow} y y x z \square z y^{s+2} x \in \mathcal{R}_{11}, \\
x y y z \square y^{s+2} z \cdots x \stackrel{x y}{\longrightarrow} x y \square y x \in \mathcal{R}_{0}, \\
x y y z \square y z y \cdots x \stackrel{x y}{\longrightarrow}(x y)^{2} z \square y z x \in \mathcal{R}_{4}, \\
x y y z \square y z z y \cdots x \stackrel{x y}{\longrightarrow}(x y)^{2} z \square y z z x \in \mathcal{R}_{7}, \\
x y y z \square y z^{r+3} y \cdots x \stackrel{x y}{\longrightarrow}(x y)^{2} z \square y z^{r+3} x \in \mathcal{R}_{15} .
\end{gathered}
$$

Proposition 28. $\mathcal{R}_{17}(\psi)=\left\{x^{m+2} y \varphi(x, y) z \square z y y z \psi(y, z) x\right\}$, where $\psi$ is not of the form $(y z)^{k} y$ if $\varphi$ is empty, is a singleton realm escaped by

$$
\begin{gathered}
x^{m+2} y \varphi(x, y) z \square z y y z \psi(y, z) x \stackrel{z x}{\longrightarrow} x(z x)^{m+1} y \square y y z \psi(y, z) z x \in\left(\mathcal{R}_{13} \cup \mathcal{R}_{14}\right)(y \leftrightarrow z), \\
x^{m+2} y \varphi(x, y) z \square z y y z \psi(y, z) x \stackrel{x z}{\longrightarrow} x^{m+1} y x z \square z y y x \in\left(\mathcal{R}_{7} \cup \mathcal{R}_{15} \cup \mathcal{R}_{16}\right)(x \leftrightarrow z) .
\end{gathered}
$$

Note that in Proposition 28 the condition stipulated for $\psi$ make $\mathcal{R}_{17}(\varphi)$ disjoint from $\mathcal{R}_{7} \cup \mathcal{R}_{15}$. 
Proposition 29. Every $y$-cap of the form $x^{m+1} y x \varphi(x, y) z \square y z^{s+2} x$, where $\varphi \neq$ $x^{k} y$ if $m=0$, defines the realm

$$
\mathcal{R}_{18}(m, s, \varphi)=\left\{x^{m+1} y x \varphi\left(x, x^{k} y\right) z \square y z^{s+2} x \mid k \geq 0\right\}
$$

which is an infinite $y$-path if $\varphi$ contains $y$ and a $y$-loop otherwise, and is escaped by

$$
x^{m+1} y x \varphi\left(x, x^{k} y\right) z \square y z^{s+2} x \stackrel{y x}{\longrightarrow}(x y)^{m+1} y x \varphi\left(y x,(y x)^{k} y\right) z \square z^{s+2} y x, k \geq 0,
$$

to $\left(\mathcal{R}_{17} \cup \mathcal{R}_{17}\right)(x \leftrightarrow z)$.

Note that in Proposition 29 the conditions stipulated for $\varphi$ make $\mathcal{R}_{18}(\varphi)$ disjoint from $\mathcal{R}_{7} \cup \mathcal{R}_{15}$.

Proposition 30. $\mathcal{R}_{19}(n)=\left\{x y x z \square z^{n+3} y \psi(y, z) x\right\}$ is a singleton realm escaped by

$$
\begin{gathered}
x y x z \square z^{n+3} y \psi(y, z) x \stackrel{z x}{\longrightarrow} x y z \square z^{n+2} y \psi(y, z) z x \in \mathcal{R}_{6} \cup \mathcal{R}_{8} \cup \mathcal{R}_{9}, \\
x y x z \square z^{n+3} y \psi(y, z) x \stackrel{x z}{\longrightarrow} y x x z \square z(x z)^{n+2} y \in \mathcal{R}_{16}(x \leftrightarrow y) .
\end{gathered}
$$

Proposition 31. $\mathcal{R}_{20}(m, \sigma)=\mathcal{C}\left(x y^{m+3} z \square \sigma(y, z) x\right)$, where $|\sigma|_{z} \geq 2$, is an $x$-cycle escaped by the arrows of the form

$$
\begin{gathered}
x y^{m+3} z \square z^{r+3} y \cdots x \stackrel{x z}{\longrightarrow} y^{m+3} x z \square z(x z)^{r+2} y \in \mathcal{R}_{14}(x \leftrightarrow y), \\
x y^{m+3} z \square z z y \cdots x \stackrel{x z}{\longrightarrow} y^{m+3} x z \square z x z y \in \mathcal{R}_{19}(x \rightarrow z \rightarrow y \rightarrow x), \\
x y^{m+3} z \square z y^{s+1} z \cdots x \stackrel{x z}{\longrightarrow} y^{m+3} x z \square z y^{s+1} x \in \mathcal{R}_{11} \cup \mathcal{R}_{11}(x \leftrightarrow z), \\
x y^{m+3} z \square y^{s+2} z \cdots x \stackrel{x y}{\longrightarrow} x y \square y x \in \mathcal{R}_{0}, \\
x y^{m+3} z \square y z y \cdots x \stackrel{x y}{\longrightarrow}(x y)^{m+3} z \square y z x \in \mathcal{R}_{10}, \\
x y^{m+3} z \square y z^{r+2} \cdots x \stackrel{x y}{\longrightarrow}(x y)^{m+3} z \square y z^{s+2} x \in \mathcal{R}_{18} .
\end{gathered}
$$

Proposition 32. $\mathcal{R}_{21}(r, s, \varphi, \psi)=\left\{x y^{r+2} x \varphi(x, y) z \square z y^{s+1} z \psi(y, z) x\right\}$ is a singleton realm escaped by

$$
x y^{r+2} x \varphi(x, y) z \square z y^{s+1} z \psi(y, z) x \stackrel{z x}{\longrightarrow} x y^{r+2} z \square y^{s+1} z \psi(y, z) z x
$$

to $\mathcal{R}_{16} \cup \mathcal{R}_{20}$ if $s>0$ or $\psi$ contains $y$, else to $\mathcal{R}_{7} \cup \mathcal{R}_{15} \cup \mathcal{R}_{20}$, and by

$$
x y^{r+2} x \varphi(x, y) z \square z y^{s+1} z \psi(y, z) x \stackrel{x z}{\longrightarrow} y^{r+2} x \varphi(x, y) x z \square z y^{s+1} x
$$

to $\left(\mathcal{R}_{6} \cup \mathcal{R}_{9} \cup \mathcal{R}_{16} \cup \mathcal{R}_{20}\right)(x \leftrightarrow z)$. 
Proposition 33. $\mathcal{R}_{22}(t, \varphi, \psi)=\left\{x y x x \varphi(x, y) z \square z^{t+2} y \psi(y, z) x\right\}$ is a singleton realm escaped by

$$
x y x x \varphi(x, y) z \square z^{t+2} y \psi(y, z) x \stackrel{z x}{\longrightarrow} x y z \square z^{t+1} y \psi(y, z) z x
$$

to $\mathcal{R}_{5} \cup \mathcal{R}_{6} \cup \mathcal{R}_{8} \cup \mathcal{R}_{9}$, and by

$$
x y x x \varphi(x, y) z \square z^{t+2} y \psi(y, z) x \stackrel{x z}{\longrightarrow} y x x \varphi(x, y) x z \square z(x z)^{t+1} y
$$

to $\left(\mathcal{R}_{20} \cup \mathcal{R}_{21}\right)(x \leftrightarrow y)$.

Proposition 34. $\mathcal{R}_{23}(m, r, \varphi, \psi)=\left\{x^{m+2} y \varphi(x, y) z \square z y^{r+3} z \psi(y, z) x\right\}$ is a singleton realm escaped by

$$
\begin{gathered}
x^{m+2} y \varphi(x, y) z \square z y^{r+3} z \psi(y, z) x \stackrel{z x}{\longrightarrow} x(z x)^{m+1} y \square y^{r+3} z \psi(y, z) z x \in\left(\mathcal{R}_{14} \cup \mathcal{R}_{19}\right)(y \leftrightarrow z), \\
x^{m+2} y \varphi(x, y) z \square z y^{r+3} z \psi(y, z) x \stackrel{x z}{\longrightarrow} x^{m+1} y \varphi(x, y) x z \square z y^{r+3} x \in \mathcal{R}_{20}(x \leftrightarrow z) .
\end{gathered}
$$

Proposition 35. $\mathcal{R}_{24}(m, s, \varphi)=\left\{x^{m+1} y y \varphi(x, y) z \square y z^{s+2} x \mid k \geq 0\right\}$, where $\varphi$ contains $x$ if $m=0$, is a singleton realm escaped by

$$
\begin{gathered}
x^{m+1} y y \varphi(x, y) z \square y z^{s+2} x \stackrel{y x}{\longrightarrow}(x y)^{m+1} y \varphi(y x, y) z \square z^{s+2} y x \in \mathcal{R}_{17} \cup \mathcal{R}_{23}, \\
x^{m+1} y y \varphi(x, y) z \square y z^{s+2} x \stackrel{x y}{\longrightarrow} x^{m+1} y x y \varphi(x, x y) z \square y z^{s+2} x \in \mathcal{R}_{18} .
\end{gathered}
$$

Proposition 36. $\mathcal{R}_{25}(m, s, \varphi, \psi)=\left\{x^{m+1} y \varphi(x, y) z \square y z^{s+1} y \psi(y, z) x\right\}$, where $\varphi$ contains $x$ if $m=0$, is a singleton realm escaped by

$$
x^{m+1} y \varphi(x, y) z \square y z^{s+1} y \psi(y, z) x \stackrel{y x}{\longrightarrow}(x y)^{m+1} \varphi(y x, y) z \square z^{s+1} y \psi(y, z) y x
$$

to $\mathcal{R}_{6} \cup \mathcal{R}_{9} \cup \mathcal{R}_{12} \cup \mathcal{R}_{14} \cup \mathcal{R}_{16} \cup \mathcal{R}_{17} \cup \mathcal{R}_{20} \cup \mathcal{R}_{21} \cup \mathcal{R}_{23}$, and by

$$
x^{m+1} y \varphi(x, y) z \square y z^{s+1} y \psi(y, z) x \stackrel{x y}{\longrightarrow} x^{m+1} y \varphi(x, x y) z \square y z^{s+1} x
$$

to $\mathcal{R}_{10} \cup \mathcal{R}_{18}$.

Proposition 37. $\mathcal{R}_{26+r}(m, r, \varphi, \psi)=\left\{x^{m+1} y \varphi(x, y) z \square y^{r+2} z \psi(y, z) x\right\}$, where $\varphi$ contains $x$ if $m=0$, is a singleton realm escaped by

$$
x^{m+1} y \varphi(x, y) z \square y^{r+2} z \psi(y, z) x \stackrel{y x}{\longrightarrow}(x y)^{m+1} \varphi(y x, y) z \square y^{r+1} z \psi(y, z) y x
$$

to $\mathcal{R}_{25}$ if $r=0$, else to $\mathcal{R}_{26+r-1}$, and by

$$
x^{m+1} y \varphi(x, y) z \square y^{r+2} z \psi(y, z) x \stackrel{x y}{\longrightarrow} x^{m+1} y \square y x \in \mathcal{R}_{0} \cup \mathcal{R}_{1} .
$$

The following realm forms are indexed from the first infinite ordinal $\omega$ on. 
Proposition 38. $\mathcal{R}_{\omega+s}(m, s, \varphi, \psi)=\left\{x^{m+2} y \varphi(x, y) z \square z^{s+2} y \psi(y, z) x\right\}$ is a singleton realm escaped by

$$
x^{m+2} y \varphi(x, y) z \square z^{s+2} y \psi(y, z) x \stackrel{z x}{\longrightarrow} x(z x)^{m+1} y \square z^{s+1} y \psi(y, z) z x
$$

to $\mathcal{R}_{25}(y \leftrightarrow z)$ if $s=0$, else to $\mathcal{R}_{26+s-1}(y \leftrightarrow z)$, and by its $(x \leftrightarrow z)$-image.

Here we stop introducing new realms and sum up what we have done. Let us call the realms with the indices $3,4,5,6,7,8,9,11,15,16,20$, together with their isomorphic copies, the realms of the first kind and all the remaining ones the realms of the second kind.

Theorem 4.1. The realms of the first kind are non-trivial strong components (i.e. containing at least two caps) covering all caps with the first term of the form $x y^{m+1} z$, hence by the symmetry $(x \leftrightarrow z)$ also all $z$-caps with the second term $z y^{m+1} x$, and all caps of the form

$$
x y x^{k+1} y z \square y z^{s+1} x \text { or } x y(y x)^{k+1} y z \square z^{s+1} y x,
$$

where $0 \leq s \leq 1$, contained in $\mathcal{R}_{4}$ for $s=0$ and in $\mathcal{R}_{7}$ for $s=0$.

The realms of the second kind are unions of singleton strong components and they cover all the remaining of caps.

Proof. For $\left[E_{m, n}\right]=x y^{m+1} z \square z^{r} y z^{s} x$, where $r+s=n \geq 1$, we have $\left[E_{0,1}\right] \in \mathcal{R}_{3}$, $\left[E_{0,2}\right] \in \mathcal{R}_{5}$, and $\left[E_{0, n}\right] \in \mathcal{R}_{8}$ for $n \geq 3,\left[E_{1,1}\right] \in \mathcal{R}_{4},\left[E_{1,2}\right] \in \mathcal{R}_{7}$, and $\left[E_{1, n}\right] \in \mathcal{R}_{15}$ for $n \geq 3,\left[E_{m, 1}\right] \in \mathcal{R}_{11}(x \leftrightarrow z)$ for $m \geq 2$, and $\left[E_{m, n}\right] \in \mathcal{R}_{20}$ for $m, n \geq 2$.

For $[E]_{m, \sigma}=x y^{m+1} z \square \sigma(y, z) x$, where $|\sigma|_{y} \geq 2$, we have $[E]_{0, \sigma} \in \mathcal{R}_{4}$ if $\sigma \sim$ $(y z)^{k+1} y$ and $[E]_{0, \sigma} \in \mathcal{R}_{6} \cup \mathcal{R}_{9}$ otherwise, $[E]_{0, \sigma} \in \mathcal{R}_{11} \cup \mathcal{R}_{16}$, and $[E]_{m, \sigma} \in \mathcal{R}_{20}$ for $m \geq 2$.

The $y$-caps with the first term non linear in $x$ are covered by the realms with the indices 10, 18, 24, 25, and $26+r$ for $r \geq 0$.

The $z$-caps with the first term non linear in $x$ and the second one non linear in $z$ are covered by the realms with the indices $12,13,14,17,19,21,22,23$, and $\omega+s$ for $s \geq 0$, and by their $(x \leftrightarrow z)$-copies.

\section{Parametrization of Path traces}

For our pourposes, we can define a parametrization of a set $S$ as a calculable surjective function $\Phi: D \rightarrow S$ whose domain is one of the sets defined recursively by stipulating that

- the set $\mathbb{N}$ of naturals is a domain;

- if $C, D$ are domains then $C \times D$ and $D^{*}$ are domains.

The number of stars intervening in the construction of the domain $D$ of $\Phi$, the star-depth of $D$, is a sort of complexity measure of the parametrization.

Let $\mathcal{S}$ be a subgraph of $\left[\mathcal{E}_{\mathcal{P}}\right]$, and let $\left[E_{0}\right],[E],[F] \in \mathcal{S}$. Denote by

- $\operatorname{Tr}_{\mathcal{S}}([E],[F])$ the set of the traces of the $\mathcal{S}$-paths from $[E]$ to $[F]$; 
- $\operatorname{Tr}_{\mathcal{S}}^{(0)}([E],[F])$ the set of the traces of the $\mathcal{S}$-paths from $[E]$ to $[F]$ with all intermediate vertices different from $\left[E_{0}\right]$;

- $\operatorname{Tr}_{\mathcal{S}}([E],-)$ the set of the traces of the $\mathcal{S}$-paths from $[E]$ to some cap of $\mathcal{S}$. As a rule, the subscript $\mathcal{S}$ will be suppressed when clear from the context (e.g. when it denotes a realm currently dealt with).

Lemma 1. We have the relation

$$
\operatorname{Tr}([E],[F])=\operatorname{Tr}^{(0)}([E],[F]) \cup \operatorname{Tr}^{(0)}\left([E],\left[E_{0}\right]\right) \operatorname{Tr}\left(\left[E_{0}\right],\left[E_{0}\right]\right) \operatorname{Tr}^{(0)}\left(\left[E_{0}\right],[F]\right),
$$

where $\operatorname{Tr}\left(\left[E_{0}\right],\left[E_{0}\right]\right)$ is a monoid generated by $\operatorname{Tr}(0)\left(\left[E_{0}\right],\left[E_{0}\right]\right)$ (possibly reduced to $\{\Delta\}$, for the identity substitution $\Delta)$.

For a realm $\mathcal{R}$ and a cap $[E] \in \mathcal{R}$, every escape path from $\mathcal{R}$ starting at $[E]$ has a beginning (initial segment) in $\operatorname{Tr}_{\mathcal{R}}([E],-)$.

For most of the realms described in the preceding section, the parametrization of $\operatorname{Tr}^{(0)}([E],[F])$, for $\left[E_{0}\right]$ suitably chosen and $[E] \neq[F]$, poses no problem and will be left to the reader. We will concentrate on the parametrization of $\operatorname{Tr}^{(0)}\left(\left[E_{0}\right],\left[E_{0}\right]\right)$, easily convertible into a parametrization of $\operatorname{Tr}\left(\left[E_{0}\right],\left[E_{0}\right]\right)$.

Proposition 39. Every realm $\mathcal{R}_{\alpha}$ with $\alpha \in\{12,13,14,17,19,21,22,23,25\} \cup$ $\{26+r \mid r \geq 0\} \cup\{\omega+s \mid s \geq 0\}$ is formed by a single cap $\left[E_{0}\right]$ with $\operatorname{Tr}\left(\left[E_{0}\right],\left[E_{0}\right]\right)=$ $\{\Delta\}$.

Proposition 40. Every realm $\mathcal{R}_{\alpha}$ with $\alpha \in\{1,10,18,24\}$ is either a $y$-loop or an infinite $y$-path and we have

$$
\operatorname{Tr}([E],-)=\left\{\left(y \mapsto x^{k} y\right) \mid k \geq 0\right\}
$$

for every $[E] \in \mathcal{R}$.

Proposition 41. Every realm $\mathcal{R}_{\alpha}$ with $\alpha \in\{6,9,16,20\}$ is, for some $m \in \mathbb{N}$ and $w \in\{y, z\}^{*}$, an $x$-cycle of the form

$$
\left\{\left[E_{i}\right]=x y^{m+1} z \square v_{i} u_{i} x\left|w=u_{i} v_{i},\right| u_{i}|=i, 0 \leq i<| w \mid-1\right\},
$$

parametrized by

$$
\begin{array}{llr}
\operatorname{Tr}^{(0)}\left(\left[E_{i}\right],\left[E_{0}\right]\right) & =\left\{\left(x \mapsto v_{i} x\right)\right\} & \text { for } i \geq 1, \\
\operatorname{Tr}\left(\left[E_{0}\right],\left[E_{0}\right]\right) & =\left\{\left(x \mapsto w^{k} x\right) \mid k \geq 0\right\} & \\
\operatorname{Tr}^{(0)}\left(\left[E_{0}\right],\left[E_{j}\right]\right) & =\left\{\left(x \mapsto u_{j} x\right)\right\} & \text { for } j \geq 1, \\
\operatorname{Tr}^{(0)}\left(\left[E_{i}\right],\left[E_{j}\right]\right) & =\{(x \mapsto t x)\} & \text { for } u_{j}=u_{i} t, \\
\operatorname{Tr}^{(0)}\left(\left[E_{i}\right],\left[E_{j}\right]\right) & =\emptyset & \text { for } i>j \geq 1 .
\end{array}
$$

Consequently,

$$
\operatorname{Tr}\left(\left[E_{i}\right],\left[E_{j}\right]\right)=\left\{\left(x \mapsto v_{i} w^{k} u_{j}\right) \mid k \geq 0\right\} \cup \operatorname{Tr}^{(0)}\left(\left[E_{i}\right],\left[E_{j}\right]\right) .
$$


Lemma 2. A monoid $M$ generated by a pair of substitutions $(x \mapsto y v x)$ and $(y \mapsto x u y)$, where $x, y \notin$ alph $(u v)$, can be parametrized by the homomorphism

$$
\Phi_{x u, y v}:(\mathbb{N} \times \mathbb{N})^{*} \rightarrow M:(m, n) \mapsto(x \mapsto y v x)^{m}(y \mapsto x u y)^{n} .
$$

Proposition 42. Every realm $\mathcal{R}_{\alpha}$ with $\alpha \in\{8,15\}$ contains, for some $m \in \mathbb{N}$ $m \in\{0,1\}$ and $n \in \mathbb{N}$, the cap $\left[E_{0}\right]=x y^{m+1} z \square y z^{n+3} x$ an $x$-cycle of the form for which

$$
\operatorname{Tr}^{(0)}\left(\left[E_{0}\right],\left[E_{0}\right]\right)=\left\{\left(x \mapsto y z^{n+3} x\right),(y \mapsto x y)\right\}
$$

hence

$$
\operatorname{Tr}\left(\left[E_{0}\right],\left[E_{0}\right]\right)=\Phi_{x, y z^{n+3}}\left((\mathbb{N} \times \mathbb{N})^{*}\right) .
$$

Proposition 43. The realm $\mathcal{R}_{11}(r, s), r \leq s, r+s \geq 2$, is formed of an $x$-cycle and of an $z$-cycle meeting at $\left[E_{0}\right]=x y^{r+1} z \square z y^{s+1} x$, hence

$$
\begin{gathered}
\operatorname{Tr}^{(0)}\left(\left[E_{0}\right],\left[E_{0}\right]\right)=\left\{\left(x \mapsto z y^{s+1} x\right),\left(z \mapsto x y^{r+1} z\right)\right\} \\
\operatorname{Tr}\left(\left[E_{0}\right],\left[E_{0}\right]\right)=\Phi_{x y^{r+1}}, z y^{s+1}\left((\mathbb{N} \times \mathbb{N})^{*}\right) .
\end{gathered}
$$

Proposition 44. The realm $\mathcal{R}_{5}$ is divided into two disjoint parts, an $x$-cycle $\mathcal{H}$ with a $y$-loop at $\left[E_{0}\right]=x y z \square y z z x$ and an $y$-cycle $\mathcal{H}(x \leftrightarrow y)$ with a $y$-loop at $\left[F_{0}\right]=y x z \square x z z y$, connected by two $z$-arrows in such a way that

$$
\begin{gathered}
\operatorname{Tr}^{(0)}\left(\left[E_{0}\right],\left[F_{0}\right]\right)=\left\{g_{x, y}=(x \mapsto y x)(z \mapsto x z)(y \mapsto z y)\right\}, \\
\operatorname{Tr}^{(0)}\left(\left[F_{0}\right],\left[E_{0}\right]\right)=\left\{g_{y, x}=(x \leftrightarrow y) g_{x, y}(x \leftrightarrow y)\right\} .
\end{gathered}
$$

Consequently, we have the parametrization homomorphisms

$$
\Phi_{y, x z z}:(\mathbb{N} \times \mathbb{N})^{*} \rightarrow \operatorname{Tr}_{\mathcal{H}}\left(\left[E_{0}\right],\left[E_{0}\right]\right)
$$

and

$\left.\Psi:\left((\mathbb{N} \times \mathbb{N})^{*} \times(\mathbb{N} \times \mathbb{N})^{*}\right)\right)^{*} \rightarrow \operatorname{Tr}\left(\left[E_{0}\right],\left[E_{0}\right]\right):(\boldsymbol{m}, \boldsymbol{n}) \mapsto \Phi_{x, y z z}(\boldsymbol{m}) g_{x, y} \Phi_{y, x z z}(\boldsymbol{n}) g_{y, x}$ (where $\boldsymbol{m}$ and $\boldsymbol{n}$ are strings of pairs of naturals).

Proposition 45. The realm $\mathcal{R}_{7}$ is divided into two disjoint parts $\mathcal{H}$ and $\mathcal{H}(x \leftrightarrow$ $y)$, containing the caps $\left[E_{0}\right]=x y y z \square y z z x$ and $\left[F_{0}\right]=x z z y \square z y y x$, connected in such a way that

$$
\begin{gathered}
\operatorname{Tr}^{(0)}\left(\left[E_{0}\right],\left[F_{0}\right]\right)=\left\{h_{x, y}=(x \mapsto y x)(z \mapsto x z)(y \mapsto z y)(x \mapsto y x)\right\}, \\
\operatorname{Tr}^{(0)}\left(\left[F_{0}\right],\left[E_{0}\right]\right)=\left\{h_{y, x}=(y \leftrightarrow z) h_{x, y}(y \leftrightarrow z)\right\}
\end{gathered}
$$

Consequently, we have the parametrization homomorphisms

$$
\Phi_{y, x z z}:(\mathbb{N} \times \mathbb{N})^{*} \rightarrow \operatorname{Tr}_{\mathcal{H}}\left(\left[E_{0}\right],\left[E_{0}\right]\right)
$$


and

$\left.\Psi:\left((\mathbb{N} \times \mathbb{N})^{*} \times(\mathbb{N} \times \mathbb{N})^{*}\right)\right)^{*} \rightarrow \operatorname{Tr}\left(\left[E_{0}\right],\left[E_{0}\right]\right):(\boldsymbol{m}, \boldsymbol{n}) \mapsto \Phi_{x, y z z}(\boldsymbol{m}) h_{x, y} \Phi_{y, x z z}(\boldsymbol{n}) h_{y, x}$

(where $\boldsymbol{m}$ and $\boldsymbol{n}$ are strings of pairs of naturals).

Proposition 46. For the cap $\left[E_{0}\right]=x y y z \square y z z x$ of $\mathcal{R}_{4}$ we have

$$
\operatorname{Tr}^{(0)}\left(\left[E_{0}\right],\left[E_{0}\right]\right)=\left\{f_{k, m}=\left(y \mapsto x^{k} y\right)(x \mapsto y x)\left(z \mapsto\left(x y(y x)^{k} y\right)^{m} z\right)(x \mapsto z x)\right\},
$$

whence the parametrization homomorphism

$$
\Omega:(\mathbb{N} \times \mathbb{N})^{*} \rightarrow \operatorname{Tr}\left(\left[E_{0}\right],\left[E_{0}\right]\right):(k, m) \mapsto f_{k, m} .
$$

We come to a conclusion. Let $E$ be an equation with $[E]$ in three cap variables, and let $\mathcal{R}[E]$ denote the realm of caps reachable from $[E]$ in $\mathcal{E}_{P}$.

The parametrizations obtained in Proposition 39-46 can be combined, for any $[F] \in \mathcal{R}[E]$, into a (cascade composite) parametrization of $\operatorname{Tr}([E],[F])$. By Proposition 11 , the family

$$
\mathcal{F}=\bigcup\{\operatorname{Tr}([E],[F])(x \mapsto 1) \mid[F] \in \mathcal{R}[E], x \in \operatorname{pivot}(F)\}
$$

is universal for $E$. Moreover, every $E f$ involves less variables than $E$, hence $\mathcal{F}$ operates a reduction of $E$ to a parametric family of simpler equations. The fact that $\mathcal{F}$ is parametric is crucial for the treatment of the family of equations $\{E f \mid f \in \mathcal{F}\}$. The latter should be divided into caps and the caps treated by the method we have outlined here.

Of course, the work we have done here for the caps in three variables must be continued, in order to see whether the parametrization for the caps in four and more variables goes as smoothly. The goal is to get a general theorem on the existence of an algorithm calculating a parametrization of a universal family of solutions for an arbitrary equation (with or without constants). Such an algorithm will certainly be rather complex to be of practical use. However, it may help establish decidability results concerning various properties of solutions to a given equation.

\section{REFERENCES}

[1] J. Jaffar, Minimal and Complete Word Unification. J. ACM 37 (1990) 47-85.

[2] Yu.I. Hmelevskiǔ, Equations in free semigroups. Trudy Mat. Inst. Steklova 107 (1971); English Translation in Proc. Steklov Inst. Math. 107 (1971) 1976.

[3] A. Lentin, Équations dans les monö̈des libres. Gautier-Villars, Paris (1972).

[4] M. Lothaire, Combinatorics on Words. Addison-Wesley (1983).

[5] G.S. Makanin, The problem of solvability of equations in a free semigroup. Mat. Sbornik 103 (1977) 147-236 (in Russian); English Translation in Math. USSR Sbornik 32 (1977) 128-198. 
[6] G.S. Makanin, On general solution of equations in free semigroups, in Proc. of IWWERT'91, edited by H. Abdulrab and J.P. Pécuchet. Springer, Lecture Notes in Comput. Sci. 677, 1-5.

[7] G. Plotkin, Building-in Equational Theories. Machine intelligence 7 (1972) 73-90.

Communicated by Ch. Choffrut.

Received May, 2000. Accepted October, 2001. 